\section{Inducing Natural Group Identity: A RDP Analysis}

\section{by Daniel John Zizzo*}

* School of Economics and Centre for Behavioural and Experimental Social Science, University of East Anglia

\begin{abstract}
A relevance, distinctiveness and plausibility (RDP) analysis is a conceptual framework that can be used to identify when potential confounds are a problem for interpreting experimental results. We illustrate this analysis using the creation or enhancement of natural group identity by the means of priming manipulations as employed in the experiments of five target papers. Such priming manipulations may lead to experimenter demand effects and may spuriously induce behavior change. Using a RDP analysis, we show how these potential confounds are likely to be problematic for all but one of the target papers.
\end{abstract}

\section{JEL classification codes}

B41, C90, D03

\section{Keywords}

Confounds, objectives, economic experiments, group identity, natural groups, priming.
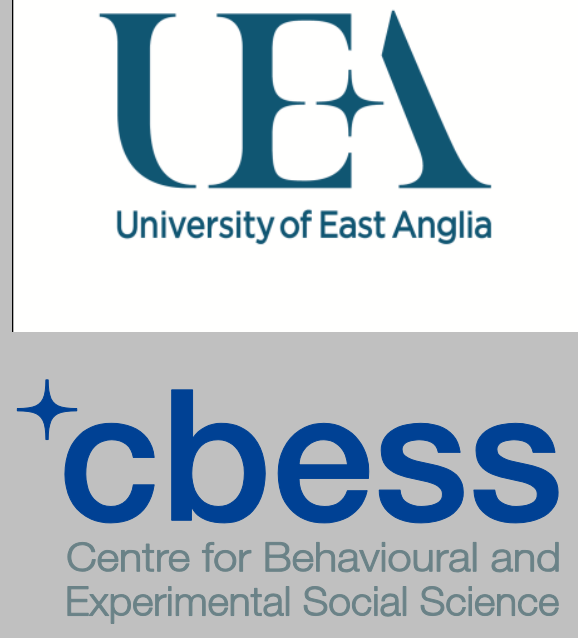

Centre for Behavioural and Experimental Social Science University of East Anglia Norwich Research Park Norwich NR4 7TJ United Kingdom www.uea.ac.uk/ssf/cbess 


\title{
Inducing Natural Group Identity: A RDP Analysis
}

\author{
Daniel John Zizzo* \\ School of Economics and CBESS \\ Norwich Research Park \\ University of East Anglia \\ Norwich NR4 7TJ \\ United Kingdom \\ d.zizzo@uea.ac.uk
}

January 2012

\begin{abstract}
A relevance, distinctiveness and plausibility (RDP) analysis is a conceptual framework that can be used to identify when potential confounds are a problem for interpreting experimental results. We illustrate this analysis using the creation or enhancement of natural group identity by the means of priming manipulations as employed in the experiments of five target papers. Such priming manipulations may lead to experimenter demand effects and may spuriously induce behavior change. Using a RDP analysis, we show how these potential confounds are likely to be problematic for all but one of the target papers.

Keywords: confounds; objectives; economic experiments; group identity; natural groups; priming.

JEL Classification Codes: B41, C90, D03.
\end{abstract}

* Phone: +44-1603-593668; fax: +44-1603-456259. I wish to thank participants to presentations in Erfurt, Heidelberg, Jena, Munich and San Sebastian for encouragement and feedback on my general analysis of experimental confounds. The usual disclaimer applies. 


\section{Introduction}

This methodological paper briefly summarizes and then applies a relevance, distinctiveness and plausibility (RDP) analysis (Zizzo, 2011), to consider one potential experimental confound present in a growing and influential set of papers on the role of specific natural group identities for economic behavior.

There are many possible definition of experimental confounds starting from John Stuart Mill when he writes that, in an experiment, "none of the circumstances which we do know shall have effects susceptible of being confounded with those of the agents whose properties we wish to study" (Mill, 1843/2009, p. 558; for two other definitions, see Patten, 2007, and Jackson, 2008). We can state that experimental confounds are any factors that 'confound', i.e. make unclear, the interpretation of experimental results connected to a particular claim made in relation to some experimental objective. A RDP analysis provides a framework to be able to evaluate whether potential confounds are indeed a problem based on their relevance, distinctiveness and plausibility. That is, it helps us think about whether, based on these dimensions, they are actual confounds we should be worried about. Admittedly, and as noted in Zizzo (2011), the number of potential confounds can in principle be very large if not logically infinite due to Duhem-Quine type of reasoning (see Smith, 1994). While this is true in principle, in practice the number of plausible, relevant and distinctive confounds will typically be a finite list (see Guala, 2002, for a parallel argument). While the RDP analysis is simply a framework to evaluate specific confounds and experimental objectives based on the body of knowledge available at any given point in time, it is fully compatible with an error elimination process by which experimental knowledge progressively grows (e.g., Mayo, 1996, and Mayo and Spanos, 2006). Furthermore, as long as appropriate qualifications are made to the claim being made and thus to the experimental objective, a RDP analysis does not necessarily mean that such a list needs to be exhausted in order for a result to be publishable. 
As a way of introduction to the specific area of application we shall apply a RDP analysis to in this paper, consider the experiment by Benjamin et al. (2010) recently published in the American Economic Review. The stated objective of this paper is to show "the effect of ethnic, racial, and gender category norms on time and risk preferences” (Benjamin et al., 2010, p. 1914). A second, more ambitious implicit objective is that group identities of this kind can be important and therefore it is meaningful to consider their effect on time and risk preferences; that this is the case is made clear by the first sentence of the conclusion, which has the claim that "our results suggest that social identity affects fundamental economic preferences” (Benjamin et al., 2010, p. 1925). Natural group identity is either created or enhanced in the experiment by the means of a questionnaire where, for example, subjects are asked to identify their race and then reflect on living with roommates of either the same or a different race. ${ }^{1}$ This is an illustration of what in psychology is called a priming manipulation: a way of nudging behavior by raising (conscious and/or unconscious) awareness of a given dimension when the decision maker evaluates a situation (see Bargh, 2006, for a recent review of the social psychological literature on priming, with a stress on the unconscious dimension).

There are two problems - potential confounds -, with doing this and then making a claim on the importance of group identity for economic preferences. One such potential confound, which we label C1 in the rest of this paper, is that subjects are simply implicitly complying with what they expect the experimental environment to demand from them. That is, there is a potential experimenter demand effect (Zizzo, 2010). A second potential confound, which we label C2 in the rest of this paper, is more basic: if social identity really matters for economic behavior, why is

\footnotetext{
${ }^{1}$ One may say that it is not possible to create a natural group identity, since either someone belongs to a natural group or one does not. For example, one either belongs or does not belong to the natural group of people with blue eyes. However, that one can descriptively be classified as belonging to a group does not mean that such group has any psychological significance to a subject. The fact that I have blue eyes does not mean that I attach any psychological meaning to this or to the set of people with blue eyes. Because of this, if a group identity does not exist psychologically ex ante, it is possible for one to be created psychologically by the means of suitable priming.
} 
priming needed for finding behavioral differences? That is to say, the priming itself may act as a confound relative to the claim made by Benjamin et al. (2010). In a RDP framework, what we need to do is to evaluate the relevance, distinctiveness and plausibility of these two potential confounds, as applicable to this as well as in other papers. Specifically, we shall also be focusing on Hoff and Pandey (2006), Afridi et al. (2010), Chen et al. (2010) and Li et al. (2011). All of these papers use natural group priming in key treatments.

Section 2 briefly presents the RDP conceptual framework, section 3 applies this framework to the inducement of natural group identity as employed by the recent experimental research, and section 4 concludes.

\section{A RDP Framework for Evaluating Experimental Confounds}

This section briefly summarizes the key elements of the RDP framework as developed in more detail in Zizzo (2011), which also considers a number of examples. Consider an experimental objective A and a potential confound $\mathrm{B}^{2}$

Relevance. The relevance of an experimental confound lies in the functional relationship between A and B: between the experimental objective and the potential confound. Specifically, it depends on whether A and B are orthogonal, work in opposite direction or work in the same direction. B works in the same direction as A (same direction relevance) if it implies actions or outcomes that go systematically in the direction of the experimental actions or outcomes implied by the experimental objectives. This is the case of potential concern. If, conversely, A and B imply actions or outcomes that go systematically in the opposite direction (contrary relevance), then,

\footnotetext{
${ }^{2}$ We take experimental objectives as givens in this paper and so are not focused on the triangular relationship involving experiments, models and the real world, as referred to for example in Guala (2005), Jones (2008) and Sitzia and Sugden (2011). There are two qualifications. First, and as noted in Zizzo (2011), if A entails saying something about a real world domain or institution, the external validity of the experiment is important as the potential confound may affect such external validity. Second, if the potential confound turns out to be a problem based on a RDP analysis, one possible response is to modify an experimental objective.
} 
unless there is a finding of no significance in which case we do not know its source, we should not be concerned, for if there is evidence for A then this holds notwithstanding B. Similarly, if A and B are not systematically related in terms of actions or outcomes (i.e., they are orthogonal), there is no problem. A final argument to consider is one of magnifying glass relevance. It goes as follows. If B artificially implements in the laboratory the equivalent of a real world feature of the domain to which the experiment is meant to apply to, then B would help enhance external validity and thus help identify how A would operate in the relevant real world domain. We shall see an example of this in section 3.

Distinctiveness. The potential confound B needs to be distinct from the objective A in order for it to be a potential problem. We shall see a partial example of identity in section 3.

Plausibility. The potential confound B needs to have a minimum degree of plausibility in order for it to be a problem. This can rely on any available evidence, direct or indirect, or failing that, it can be based on the available evidence at a given point in time, to reach a judgment about the plausibility of B relative to objective A. As stated in Zizzo (2011), the judgments employed in a RDP evaluation of a potential confound are judgments operating at a given point in time and are not meant to preclude research which may change, even radically, those judgments in future RDP evaluations. For example, B may appear irrelevant to A but, in the light of new research, this may change; or what may be seen as plausible today may, in the light of new research, be shown to be implausible, or vice versa.

Taking stock of the analysis. The RDP analysis suggests that, if a potential confound $\mathrm{B}$ is relevant, distinctive and plausible, and relevance may not be adequately defended as magnifying glass relevance (i.e., it is problematic), then there is a problem that needs to be addressed. ${ }^{3}$

\footnotetext{
${ }^{3}$ In terms of the common dichotomy between internal validity and external validity of experiments, we could say that the confound makes the interpretation of the experimental data problematic, thus invalidating internal validity, and may also spoil the external validity of the experiment if A entails saying something about a real world domain or institution.
} 
Depending on the case, it can be addressed by re-designing the experiments; collecting more data or running additional statistical analysis; or by dropping or appropriately modifying the experimental objective A.

\section{A RDP Analysis}

We consider five target papers in our analysis: Hoff and Pandey (2006), Afridi et al. (2010), Chen et al. (2010) and Li et al. (2011) as well as Benjamin et al. (2010), which we have already reviewed in the introduction. Our goal is to verify how the experiments described in these target papers fare when a RDP analysis is applied to them in the context of two specific potential confounds, which we have labeled as C1 (experimenter demand effects) and C2 (spurious creation or enhancement of natural group identity) as they may originate from natural group category priming.

Experimental objectives and inducement of group identity. Hoff and Pandey (2006) was an experiment with Indian villages children aimed to look at the effect of stereotype threat in the context of task performance. In the key treatments, various bits of information for each child were publicly announced, including the caste, and they had to confirm this verbally. This was in the presence of other same caste status children or in the presence of mixed caste groups. Again in the context of task performance, Afridi et al. (2010) ran an experiment in Beijing with Chinese children with either high (i.e., city) or low (i.e., rural) household registration status. Their objective was "to investigate the causal impact of social identity on individuals’ response to economic incentives” (p. 2). Before undergoing a Hoff and Pandey (2006) type of manipulation of publically revealing the group each child belonged to, the children had to answer a questionnaire to prime either their city or rural identity (i.e., they were asked whether they considered themselves Beijing locals). In their natural group treatment, Li et al. (2011) again primed group natural identity by the means of a 
questionnaire, in this case comparing and contrasting one's own college major with that of others; their goal was argue for role of group identity on oligopoly market behavior. Chen et al. (2010) argued for the significance of natural identities such as being either Asian or Caucasian on cooperation in five Prisoner's Dilemma games. They again used a suitable questionnaire as a priming manipulation, and, on top of this and between rounds of play, they showed pictures of China for five second in relation to Asian subjects and pictures of Europe for five seconds in relation to Caucasian subjects. Such pictures "were pretested to establish that they primed the appropriate identities” (p. 9).

Relevance. In principle, both C1 and C2 have same direction relevance as they imply behavior that would be in the same direction as that entailed by the experimental objectives of the target papers, therefore potentially confounding the interpretation of the results. An experimenter demand (C1) would operate through enhancing behavioral conformity to expectations associated to group identity in general or with reference to norms or stereotypes associated with specific groups. Priming manipulations (C2) would operate, more fundamentally, at the level of enhancing or even creating the group identity that is alleged to be responsible for behavioral change as identified by the target papers.

A magnifying glass relevance argument could however be made in relation to Hoff and Pandey (2006). The priming, and any associated experimenter demand, could be thought of as artificially modeling cases where stereotype threat is present in the real world for Indian schoolchildren; insofar as the objective of the experiment is to identify the effects of stereotype threat, then, if we believe that such stereotype threat is a real world problem, the experimenter demand works as a magnifying glass enabling us to capture its effects.

Distinctiveness. Both C1 (experimenter demand effects) and C2 (making group identity matter through priming) are distinctive from the objectives of all five of the papers. The only potential partial exception is in the case of Hoff and Pandey (2006): the public announcements, insofar as they induce experiment demand, are a form of social pressure and per se a source of 
stereotype threat, which is the object of investigation of this paper; therefore, one could argue that there is at least a partial identity between $\mathrm{C} 1$ and the experimental objective.

Plausibility. We begin from C1, which is arguably comparatively more difficult to evaluate. It is plausible to think that public announcements by an authority to children may have led the low status children of Hoff and Pandey (2006) and Afridi et al. (2010) to feel they should take the reminder of their status seriously.

However, since Hoff and Pandey (2006) have a control treatment with same caste children and public announcement as well as a treatment with mixed caste children and public announcement, they are able to provide relevant evidence regarding the effect they are interested in versus the experimenter demand implied by the public announcement.

There is no such control treatment in Afridi et al. (2010). Furthermore, C1 is at least as plausible or probably more plausible in Afridi et al. (2010) as they combined the public announcement with a priming questionnaire.

With a university student sample, Benjamin et al. (2010) claim to have direct evidence against an experimenter demand effect interpretation of the effect of their questionnaire priming since (in a part of their experiment) they asked subjects the objective of the experiment postexperimentally and could not find support for an interpretation in terms of experimenter demand effect from that. Post-experimental questions or questionnaires of this kind are problematic, however, as what people say has often very little to do with the implicit cognitive mechanisms that underlie priming (that is, there is a dissociation between implicit and explicit knowledge: Shanks and St John 1994); if we believe that C1 holds, it would hold even more for post-experimental questions or questionnaires of this kind; and there are experimenter demand effects which are not related directly to conforming to the perceived wishes of the experimenter, but are rather purely cognitive, i.e. they simply follow from the process by which subjects employ whatever cues are given in an unfamiliar decision problem to make sense of it (Zizzo, 2010). 
The plausibility of C1 is likely to be at least as high or higher for Chen et al. (2010) than for Benjamin et al. (2010). This is true for two reasons: first, the social (interactive) dimension of Chen et al. (2010) games relative to the individual choice tasks of Benjamin et al. (2010); second, and more significantly, the presence of the pictures priming to combine and interact with the questionnaire priming.

In relation to $\mathrm{C} 2$, the analysis is more straightforward. The plausibility of the priming having an effect which otherwise would not have been obtained lies in the fact, which the authors of the target papers would not dispute, that in all of these papers it did seem to have some effect.

Pulling the analysis together. We can now summarize how the papers fare based on a RDP analysis of C1 and C2, where C2 appears to be a more unequivocal problem than C1. To repeat, in order for a potential confound to be a problem (and outside the realm of contrary relevance which is not relevant here), it needs simultaneously to satisfy conditions of same direction relevance, of distinctiveness and of some minimum threshold of plausibility.

Hoff and Pandey (2006) is the paper that appears most in the clear based on a RDP analysis. On the relevance dimension, a magnifying glass relevance argument can be employed to defend it against C1; on the distinctiveness dimension, there is at least a partial overlap between experimental objective and both $\mathrm{C} 1$ and $\mathrm{C} 2$; and on the plausibility dimension, there is evidence that appears difficult to explain in terms of C1. It is instructive to understand what makes this paper a good one to handle C1 and possibly C2 as well: it not only benefits from an extra control treatment (which helps on the plausibility dimension), but also from an appropriately qualified experimental objective (which helps on the relevance and distinctiveness dimensions).

The other papers are not equally cautious in what they claim and this contributes to C1 and C2 being problems (that is, actual confounds). It is then incumbent on researchers to think of possible responses to these problems.

One possible response that would handle both $\mathrm{C} 1$ and $\mathrm{C} 2$ would be to run additional treatments showing that there are effects of the kind predicted even without the priming 
manipulation. A less satisfactory response that would however at least help handle $\mathrm{C} 1$ would be to run treatments that provide at least some partial control for experimenter demand effects, e.g. via behavioral tasks or appropriate psychological questionnaires (for recent examples, see Hargreaves Heap and Zizzo, 2011; Zafar, 2011; or Zizzo and Fleming, 2011).

With the current data, one would need to either drop the affected experimental objectives or modify the experimental objectives to make them more congruent with what has actually been shown. Li et al. (2011) have a treatment with an artificial group manipulation rather than a natural group manipulation, and so formulating objectives in terms of natural group manipulations could be dropped entirely in favor of making the paper stand purely on the artificial group manipulation. ${ }^{4}$ In the case of Benjamin et al. (2010), the experiment does not show the effects of specific natural groups norms on time and risk preferences, let alone an effect on 'fundamental economic preferences', but, if we reformulate the experimental objective to one of how stereotype threat affects time and risk preferences for specific natural group categories, and if one buys the magnifying glass relevance argument that such stereotype threat may indeed be one that exists for people making time and risk preferences decisions in the real world the paper does provide information about this, then the paper is informative insofar as this more modest objective is concerned. In the case of Afridi et al. (2010), conditional on the presence of a possible experimenter demand effect that may or may not have magnifying glass relevance but which is expected to be stronger than in Hoff and Pandey (2006), we could again frame the experimental objective in terms of effect of a stereotype threat on behavior, though what we might say about this remains weaker than in Hoff and Pandey (2006) due to the lack of an additional treatment and the stronger nature of the priming manipulation. The especially strong nature of the manipulation in Chen et al. (2010) makes drawing inferences from this paper difficult, but whatever information there is could again be framed purely in terms of stereotype threat.

\footnotetext{
${ }^{4}$ We do not engage in a RDP analysis of artificial group manipulations in this paper.
} 


\section{Conclusions}

A relevance, distinctiveness and plausibility (RDP) analysis is a conceptual framework that can be used to identify when potential confounds may be a problem for the experimental objectives, and, if they are a problem, what are valid or less valid ways of addressing objections based on such confounds. Potential confounds are assessed against experimental objectives in terms of their relevance, distinctiveness and plausibility. We illustrated this analysis using the creation or enhancement of natural group identity by the means of priming manipulations in the experiments of five target papers. These manipulations lead to two potential experimental confounds, namely experimenter demand effects and the fact that it may spuriously enhance or even create natural group identity and thus lead to behavior change where none or little would be expected in the real world with such natural categories. Using a RDP analysis, we showed how these potential confounds are likely to be problematic for all but one of the target papers, and identified ways forward. 


\section{References}

Afridi, F., Li, S.X. and Y. Ren 2010. Social identity and inequality: the impact of China's Hukou system. University of Delhi Working paper.

Bargh, J.A. 2006. What have we been priming all these years? On the development, mechanisms, and ecology of nonconscious social behavior. European Journal of Social Psychology 36: 147-168.

Benjamin, D., Choi, J.C. and J. Strickland 2010. Social identity and preferences. American Economic Review 100: 1913-1928.

Chen, Y., Li, S.X., Liu, T.X. and M. Shih 2010. Which hat to wear? Impact of natural identities on coordination and cooperation. University of Michigan Working Paper.

Guala, F. 2002. On the scope of experiments in economics: Comments on Siakantaris. Cambridge Journal of Economics 26: 261-267.

Guala, F. 2005. The Methodology of Experimental Economics. Cambridge: Cambridge University Press.

Hargreaves Heap, S.P. and D.J. Zizzo 2011. Emotions and chat in a financial markets experiment. Social Science Research Network Discussion Paper.

Hoff, K. and P. Pandey 2006. Discrimination, social identity, and durable inequalities. American Economic Review 96: 206-211.

Jackson, S.L. 2008. Research Methods: A Modular Approach. Belmont, CA: Wadsworth.

Jiménez-Buedo, M. and L.M. Miller, 2010. Why a trade-off? The relationship between the external and internal validity of experiments. Theoria 69: 301-321.

Jones, M.K. 2008. On the autonomy of experiments in economics. Journal of Economic Methodology 15: 391-407.

Li, S.X., Dogan, K. and E. Haruvy 2011. Group identity in markets. International Journal of Industrial Organization 29: 104-115. 
Mayo, D. and A. Spanos 2006. Severe testing as a basic concept in a Neyman-Pearson philosophy of induction. British Journal of the Philosophy of Science 57: 323-357.

Mayo, D. 1996. Error and the Growth of Experimental Knowledge. Chicago: University of Chicago Press.

Mill, J.S. 1843/2009. A System of Logic, Ratiocinative and Inductive. New York: Harper \& Brothers/Project Gutenberg EBook.

Patten, M.L. 2007. Understanding Research Methods: An Overview of the Essentials. Glendale, CA: Pyrczak Publishing.

Shanks, D.R. and M.F. St John. 1994. Characteristics of dissociable human learning systems. Behavioral and Brain Sciences 17: 367-447.

Sitzia, S. and R. Sugden 2011. Implementing theoretical models in the laboratory, and what this can and cannot achieve. Journal of Economic Methodology 18: 323-343.

Smith, V.L. 1994. Economics in the laboratory. Journal of Economic Perspectives 8: 113-131.

Zafar, B. 2011. An experimental investigation of why individuals conform. European Economic Review 55: 774-798.

Zizzo, D.J. 2010. Experimenter demand effects in economic experiments. Experimental Economics 13: 75-98.

Zizzo, D.J. 2011. Confounds and objectives in economic experiments. Social Science Research Network Discussion Paper.

Zizzo, D.J. and P. Fleming 2011. Can experimental measures of sensitivity to social pressure predict public good contribution? Economics Letters 111: 239-242. 